\begin{tabular}{|c|c|c|}
\hline \multirow{2}{*}{ IDUNAS } & NATURAL \& APPLIED SCIENCES & 2019 \\
& JOURNAL & No.2 \\
$(30-37)$ \\
\hline
\end{tabular}

\title{
Comparison of CNN and SVM in detection of activation in malaria cell images
}

\author{
Research Article \\ Jale BEKTAŞ $1^{*}$ (iD) \\ I Mersin University, School of Applied Technology And Management Of Erdemli, Department of Computer Technology and \\ Information Systems, Erdemli, Mersin, TURKEY

\begin{abstract}
Author E-mail:
bektasjale@mersin.edu.tr

Tel: +903243610001

Fax: + +903243610073

*Correspondence to: J. Bektaş, Mersin University, School of Applied Technology And Management Of Erdemli, Department of Computer Technology and Information Systems, Erdemli, Mersin, TURKEY

DOI: 10.38061/idunas.632709
\end{abstract}

Received: October 14, 2019; Accepted: December 31, 2019

\section{Abstract}

Malaria is a disease caused by parasites that are transmitted through the enzymes of Anophele mosquito and cause symptoms in fatal danger. Thick and thin film microscopic examination of smears taken from blood is the most reliable method for diagnosis. In the manual examination of the smears, the expertise of the examiner and the quality of the smear significantly affect the accuracy of the diagnosis. Pattern recognition, classification techniques on blood smear images of Malaria and Malaria automatic diagnosis are among the subjects of research. In this study, Convolutional Neural Networks including InceptionV3, GoogLeNet, AlexNet, Resnet50, Vgg16 networks by using six-fold cross validation were applied and performance evaluations were performed with Support Vector Machine (SVM) which is a Machine Learning method. Support Vector Machine. It was found out that Deep Learning methods achieved at least 9.43\% of accuracy difference performance compared to SVM method based on the features of the input sample images. This difference was 0.08 for F-Score and 0.16 for Youden's index.

Keywords: classification, convolutional neural network, support vector machines, Pattern recognition

\section{INTRODUCTION}

Malaria is an infectious disease caused by the infection of erythrocytes by Plasmodium parasites, in which female anopheles mosquitoes carrying parasites inoculate humans during feeding. Since the treatment of malaria patients varies according to the detected Plasmodium species, it is extremely important to distinguish Plasmodium species in the diagnosis of malaria. The most widely used method in the microbiological diagnosis of malaria all over the world is to examine the preparations prepared by staining the blood taken from the fingertip of patient under a light microscope (Who, 2019). Thick smear and thin smear in this examination made by diagnosis of plasmodium infected or non-infected is diagnosed. When investigating the presence of parasites with thick drops, the species causing infection by fine smear is 
identified. Support can be obtained from pathology departments in terms of species separation. Malaria can be treated if appropriate diagnostic and therapeutic methods are used, but alternative solutions can be produced by machine learning Since rapid diagnostic methods are expensive and underdeveloped areas are affected by the disease alternative solutions get more attraction.

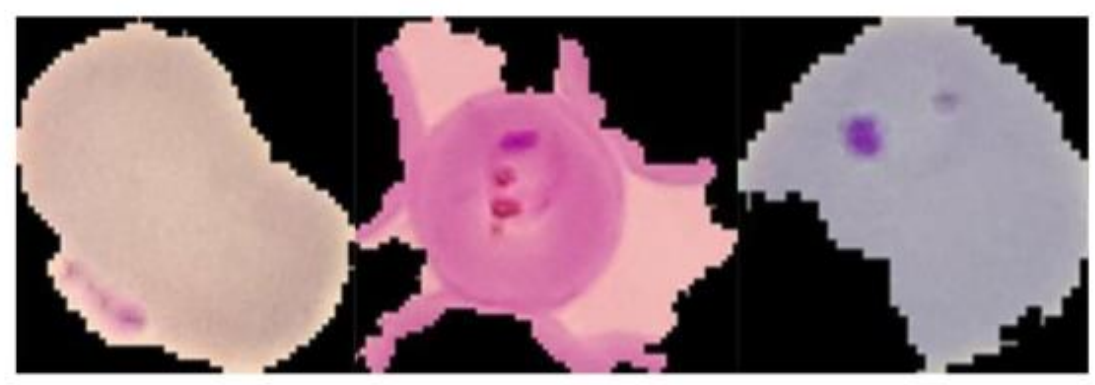

(a)

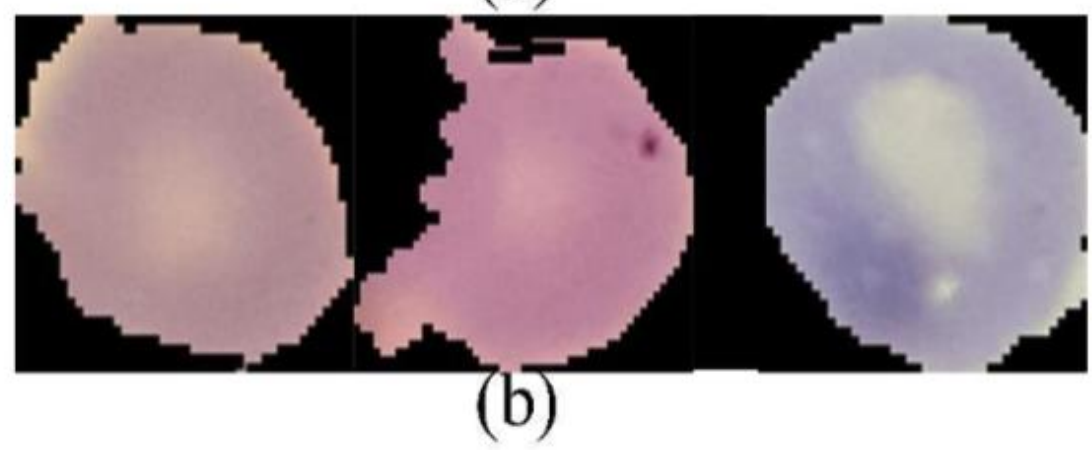

Figure 1. Red blood sample images. (a) the top three images are parasitized.(b) bottom three images are uninfected samples.

There are several studies which were applied to both thick and thin blood smear images by using machine learning methods. Support Vector Machines (SVM) and Multilayer Perceptron neural network (MLP) are used for quantification of infected erythrocytes in thin blood films as well as a precise determination of their infection stage in (Diaz, et al., 2009). Bayesian classifier and SVM are used for automated detection of malaria infected erythrocyte images using light microscopy and 84\% of accuracy is achieved with bayesian learning (Das, et al., 2013). In another study, to classify all features on malaria parasites in thin blood smear MLP method is used and achieves accuracy of 87.8\% (Nugroho, et al., 2015). Backpropagation feed forward neural network is used with image processing techniques to distinguish parasite infected images from non-infected blood images by segmenting and classfying respectively (Ahirwar, et al., 2012). The Hessian based edge detection filter is used to segment red blood cells and decision tree is used for malaria parasite classification and viability quantification (Moon, et al., 2013). Authors in (Prasad, et al., 2012) developed a decision support system for malaria parasite detection. In addition to this a mobile application has been developed which provides expert support offline.

Deep learning researchs have been a rising trendy subject with the routine methods on images and videos. The capabilities of particularly designed transfer learning paradigms, convolutional neural networks (CNN) have been very popular on pattern recognition and image classification. CNN network with 16-layers is used for analysing images for malaria diagnosis by comparing the network with AlexNet-SVM transfer learning implementation (Liang, et al., 2016). Another work compares transfer learning methods such as AlexNet, GoogleNet, LeNet with SVM and compares the performances for diagnosis of malaria (Dong, et al., 2017). Red blood cell detection and segmentation problems are examined by using malaria image 
acquisition techniques by comparing with approximately 50 image processing and classification techniques (Poostchi, et al., 2018).

In this work, five CNN networks such as AlexNet, VGG16, InceptionV3, GoogleNet, ResNet50 are used for detecting infected malaria red blood cells. The selected state-of-the-art networks are used in different studies particularly. They have different architectures of depth, and when the parameters are optimized, there is a variety in which we can measure its impact in comparison. CNN networks are compared with SVM which is one of the most popular image recognition and classification machine learning algorithm. SVM is applied by using HOG method to extract the features of the images and two kernel selection options in the classification stage are tried.

\section{EXPERIMENTAL SECTION}

\subsection{Convolutional Neural Networks}

Basically, it is a Deep Learning model that uses the standard Neural Network to solve the classification problem but uses other layers to identify information and identify certain features. Such neural networks, first performed by Yann LeCun in 1990, became widespread after 2012 (Krizhevsky, et al., 2012).

In CNNs a filter is indicated by a vector. The filter allows us to measure how much an input segment looks like a property. The image classification process takes an input image and determines the probability that it is in a predetermined class as output. RGB images such any of size 480X480 will be represented by a series of $480 \mathrm{X} 480 \mathrm{X} 3$ pixels and each number in that sequence will be identified by a number between $0-255$ representing the pixel density at that point. As a result, when training the $\mathrm{CNN}$ an image is taken, passed through a series of nonlinear, convolutional, size-reducing and fully connected layers. The output that will give a class or probability for this input is obtained (LeCun, et al., 2010). Fig.2. shows an example of the layer hierarchy and ordering in any CNN networks. Network architecture begins with an input layer and finalised with a fully connected layer. Classification is performed via Softmax. The weights on which layers should not be used and the adjustment on the output layer are explained in detail in Section 2.1.1.

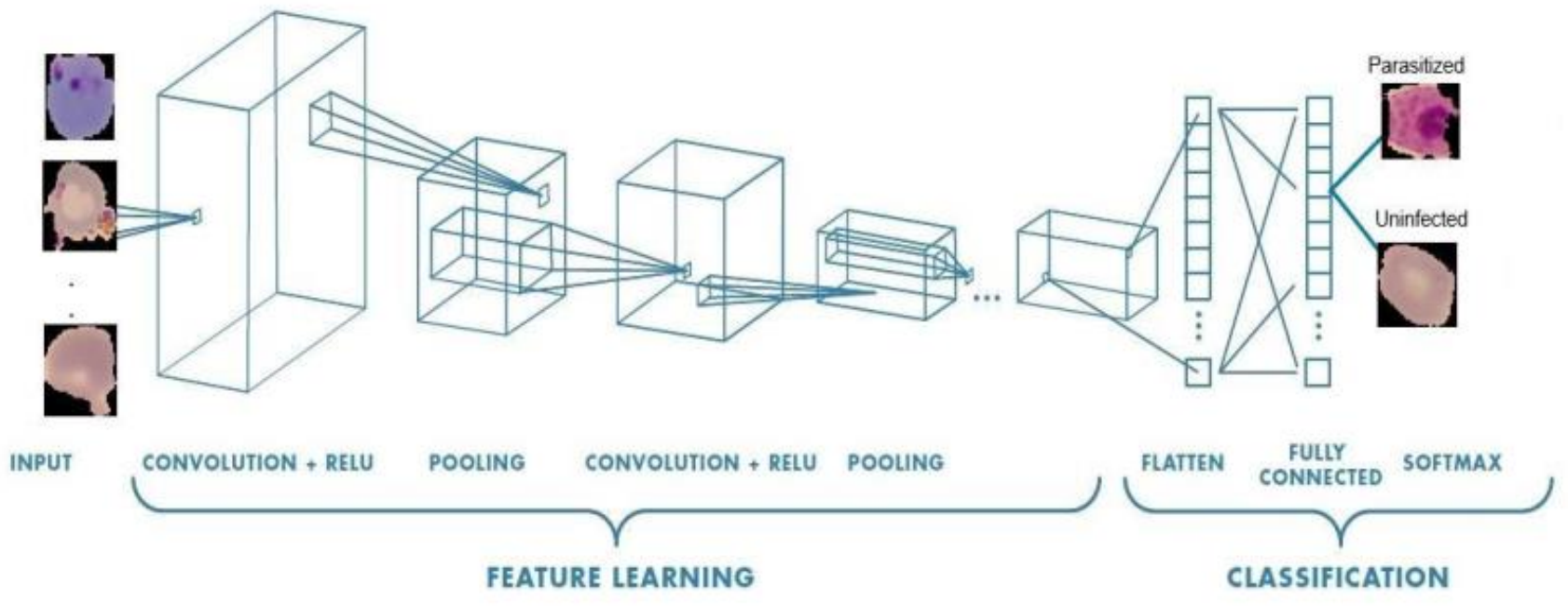

Figure 2. CNN Network Architecture

Transfer learning is a machine learning technique in which a model trained for a task is redesigned in a related second task and an optimization that provides rapid progress or improved performance in modeling is the second task. To construct a new CNN network using Malaria dataset which has the compilation of 27558 images can take up to a number of weeks. However, training the state-of-the-art networks with pretrained weights can speed up the learning process. For this reason, the use of state- of-the-art network 
architectures are practical and responsive for classification with pretrained weight values in specific layers. Some of networks which were used in this study are shown in Table 1.

Table 1. Properties of CNN Networks

\begin{tabular}{lllll}
\hline CNN Architecture & Year & Layers Deep & Input Size & Developed by \\
\hline AlexNet & 2012 & 8 & $227 \times 227$ & Alex K et al \\
VGGNet & 2014 & 16 & $224 \times 224$ & Simonyan, Zisserman \\
GoogleNet & 2014 & 22 & $224 \times 224$ & Google \\
Inception V3 & 2015 & 42 & $299 \times 299$ & Szegedy et al. \\
ResNet & 2015 & 50 & $224 \times 224$ & Kaiming He \\
\hline
\end{tabular}

\subsubsection{Parameters of Networks}

Network architectures at different depths may react differently depending on the nature of the dataset to which it is applied. Therefore, some changes must be made to the input dataset before each application and its parameters must be optimized. In this study, The Malaria image dataset is re-organized according to the image size required by the classifiers as an initial step. While input dataset pixel sizes for VGGNet and ResNet is resized to 224X224, GoogLeNet, InceptionV3 and AlexNet are resized to 227X227.

After the input image set is presented to the networks, they are progressed to the classifier layer in line with the architecture. Some arrangements are made in the architecture. In this respect:

- The Fully Connected Layer is re-arranged according to the Class Number; and the Connected Class Layer is re-arranged for Binary Output (not according to the 1000-class).

- According to the transfer learning strategy, pre-trained weight values are loaded. However, since the networks are conducted on object recognition, the weight values of the first four layers are frozen. In this way, the dominant effects for the general characteristics of the images on the network are avoided. Classification of skin lesions may be more specific only by training of the last layers.

- The connections between the layers are created again.

- 10 -6 learning rate and 0.9 momentum value is used.

- 6-fold cross validation is executed.

\subsection{Support Vector Machines (SVM)}

Linear kernel applications of SVM are very common and can be integrated with other machine learning algorithms that can result in having both good interpolation ability (Bektaş \& Ibrikçi, 2017). However, instead of making linear kernel mixture, Radial based functions (RBF) and polynomial kernel usages are considerably popular among other kernels. SVM has a very powerfull potential for approximation of kernels such as RBF and polynomial kernel functions with their estimated optimised parameters to analyze and classify image set (Kavzoglu \& Colkesen, 2009; Ring \& Eskofier, 2016). In these kernels, training samples in $\mathrm{R} \mathrm{N}$ are seperated by hyperplane which are considered to be preassigned to two classes A or B.

Mathematically notation of SVM is $K\left(x_{\mathrm{I}}, x_{J}\right)=\varphi(x) \cdot \varphi\left(x_{J}\right)$ which is identified as kernel functions. RBF is easy to apply and may be faster among all kernel functions. Whenever mathematical notation is seeming to be easy, when kernel dimension $(\gamma)$ increases the process of algorithm to be more complicated. For a RBF kernel SVM, the function is,

$$
e^{-\gamma\|(x-x i)\|^{2}}
$$


Evaluated for each $\mathrm{xi}$.

For the RBF kernel, the $C$ parameter was varied from 0.1 to 2 with increment steps of 0.1 . For the Polynomial kernel, $\mathrm{d}$ parametes is considered as important. In this study, 2 for $d$ is given for Malaria image set classification. Polynomial kernel function is given in Eq.2.

$\frac{((x . y)+1)^{d}}{\sqrt{((x . x)+1)^{d}((y \cdot y)+1)^{d}}}$

Selection of the best parameters was carried out through a 6- fold cross validation and performed for every parameter combination.

\subsubsection{HOG Feature Extraction Method}

SVM needs a feature extracture when used in image classification. In this study, Histogram of Oriented Gradients (HOG) feature extraction method is preferred because of its capabilities on object detection (Lin, et al., 2011). HOG divides an image into small square cells, calculates the histogram of directed gradients in each cell and focuses on shape information within each patch. Block-shaped pattern supports the normalization process, and finally and identifier is obtained for each cell. In experiments, the best performance of SVM classifier is obtained with 4X4 cell size of HOG. Training samples with different cell sizes are given in Figure 3.
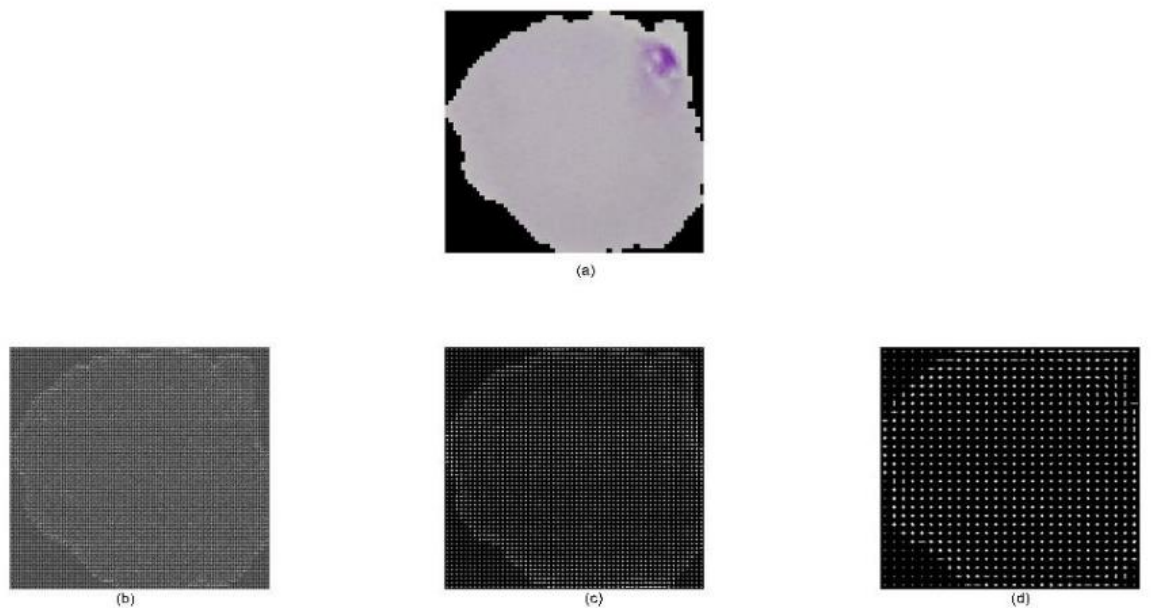

Figure 3. (a)Parasitized sample original image. (b) $2 X 2$ ell Size with 443556 training features. (c) $4 X 4$ Cell size with 108900 training samples. (d) 8 X8 Cell size with 26244 training samples.

\subsection{Malaria Cell Images Dataset Compilation for Training}

The dataset is from collections found at the National Library of Medicine and the National Institutes of Health. Whole images consist of segmented cells from the thin blood smear slide images from the Malaria Screener research activity and available from (U.S. National Library). Sample folder contains two subfolder, while Parasitized has 13779 infected thin blood smear images uninfected has 13779 images. Ratio of two classes samples is 1:1. Malaria Cell Images Dataset can be download from https://www.kaggle.com/iarunava/cell-images-for-detecting-malaria. The image compilation dataset consists of segmented images, so no preprocessing operation is performed. 


\section{RESULTS AND DISCUSSION}

The state-of-the-art networks are constructed on ImageNet dataset and based on object recognition concept. Unlike 1000-class output label structure of imagenet Dataset, the dataset used in this study has 2 classes, which required a class number adjustment in Softmax Layer.

Transfer learning is used for all five CNN's using 6-fold cross validation method. Loss functions are given for the networks in Figure 4. which have the highest performance-lowest performance. GoogleNet converged at the 20 th epoch and seems to be same. For InceptionV3, the error tends to fall until the last iteration. As the number of layers was less such as 22 layers of deep for GoogleNet, there was a possibility of overfitting depending on the number of samples being trained. No doubt larger size networks requires longer computational time, unfortunately long training-validation times may not result in good performance. We can observe this in Resnet50 results in Table 2. Although it is thenetwork with the maximum depth of 50 layers in the study, there is a ratio of 5.46 between the best results which is InceptionV3.

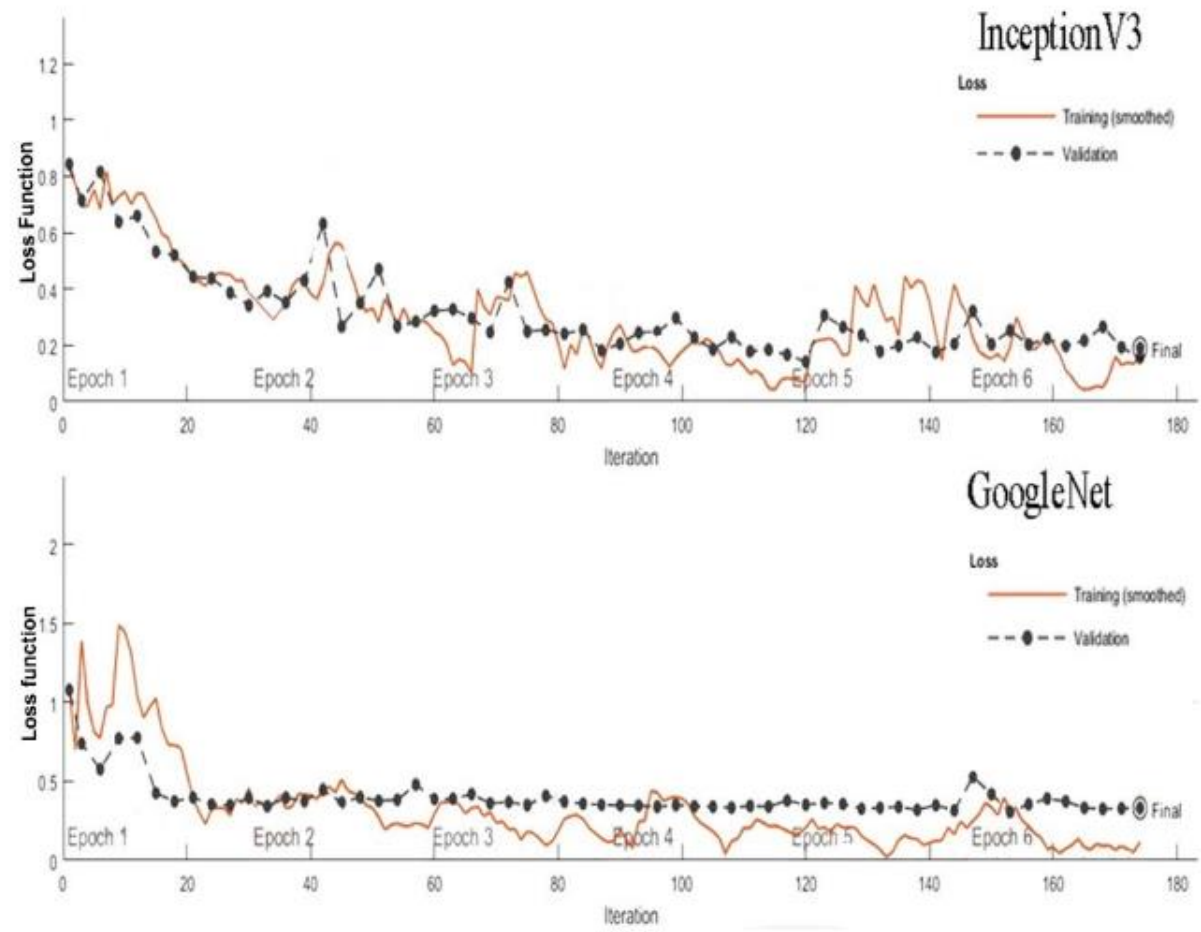

Figure 4. The loss functions of the lowest performed network GoogleNet and highest performed network InceptionV3.

After the validation, the CNNs are tested. As for the results InceptionV3 outperformed other models with the accuracy of $96.09 \%$ and the F-Score of $0.97 \%$. SVM placed the 6th and 7th with two kernel selections between other methods which is shown in Table 2. SVM with polynomial kernel outperformed by a small margin SVM with RBF kernel with about $0.4 \%$ improvement. SVM algorithm is analyzed on MATLAB environment for feature learning and classification. Nevertheless, SVM far behind the CNNs. 
Table 2. Performance metrics of individual models.

\begin{tabular}{lccllllll}
\hline Model & Accuracy & Sen & Spec & $\begin{array}{l}\text { LR } \\
+\end{array}$ & $\begin{array}{l}\text { Youden's } \\
\text { index }\end{array}$ & F-Score & ErrRate & $\begin{array}{l}\text { Process } \\
\text { Time }\end{array}$ \\
\hline Inceptionv3 & 96.09 & 0.96 & 0.92 & 12 & 0.88 & 0.97 & 0.031 & 29017 \\
$\begin{array}{l}\text { AlexNet } \\
\text { Vgg16 }\end{array}$ & 93.75 & 0.93 & 0.95 & 18.6 & 0.88 & 0.94 & 0.062 & 330 \\
& 92.97 & 0.92 & 0.94 & 15.3 & 0.86 & 0.93 & 0.07 & 2598 \\
Resnet50 & & & & 3 & & & & \\
$\begin{array}{l}\text { GoogleNet } \\
\text { SVM }\end{array}$ & 90.63 & 0.98 & 0.8 & 4.9 & 0.78 & 0.92 & 0.09 & 38439 \\
(polynomial) & 89.06 & 0.9 & 0.87 & 6.92 & 0.77 & 0.9 & 0.1 & 774 \\
SVM (RBF) & 86.50 & 0.88 & 0.84 & 5.51 & 0.72 & 0.89 & 0.14 & 4400 \\
\hline
\end{tabular}

The performance of the models are evaluated with metrics including accuracy, ACC, Sen, Spec, LR+, Youden's index, F-Score, ErrorRate, Process Time(seconds).

\section{CONCLUSION}

Many studies have been performed in the past for diagnosis over malaria cell blood images and machine learning methods have been evaluated on different image sets in each study. The dataset which is used in this study is from collections found at the National Library of Medicine and the National Institutes of Health. Whole images consist of segmented cells from the thin blood smear slide images from the Malaria Screener research activity and available from (U.S. National Library). Ratio of two classes samples is 1:1, so 1:1 ratio and 1000 samples were selected for each class and used for train. . The state-of-the-art CNN networks have different depth architectures, and when the parameters are optimized, there is a variety in which we can measure its impact in comparison. Parameters of the networks are optimized, and CNN networks are compared with SVM which is one of the most popular image recognition and classification machine learning algorithms. Simulation results showed that high recognition accuracy and F-Score could be achieved by CNN's with very small parameter adjustments. We have demonstrated that deep learning techniques have achieved better results than SVM, which is one of the best classifiers in image recognition.

\section{REFERENCES}

Ahirwar, N., Pattnaik, S., \& Acharya, B., Advanced image analysis based system for automatic detection and classification of malarial parasite in blood images. International Journal of Information Technology and Knowledge Management, 5(2012), pp.59-64.

Bektaş, J., Ibrikci, T., Hybrid classification procedure using SVM with LR on two distinctive datasets, In Proceedings of the 6th International Conference on Software and Computer Applications, Bangkok, Thailand, February 26 28, 2017, pp. 68-71, ACM.

Das, D. K., Ghosh, M., Pal, M., Maiti, A. K., \& Chakraborty, C., Machine learning approach for automated screening of malaria parasite using light microscopic images. Micron, 45(2013), pp.97-106.

Díaz, G., González, F. A., \& Romero, E., A semiautomatic method for quantification and classification of erythrocytes infected with malaria parasites in microscopic images. Journal of Biomedical Informatics, 42((2009), pp.296307.

Dong, Y., Jiang, Z., Shen, H., Pan, W. D., Williams, L. A., Reddy, V. V., et.al, Evaluations of deep convolutional neural networks for automatic identification of malaria infected cells, In 2017 IEEE EMBS International Conference on 
Biomedical \& Health Informatics (BHI), 16-19 Feb. 2017, Orlando, FL, USA, pp. 101-104, IEEE.

Kavzoglu, T., \& Colkesen, I., A kernel functions analysis for support vector machines for land cover classification. International Journal of Applied Earth Observation and Geoinformation, 11(2009), pp.352-359.

Krizhevsky, A., Sutskever, I., Hinton, G. E., Imagenet classification with deep convolutional neural networks, In Advances in neural information processing systems, (2012), pp.10971105 .

LeCun, Y., Kavukcuoglu, K., Farabet, C., Convolutional networks and applications in vision, In Proceedings of 2010 IEEE International Symposium on Circuits and Systems, 30 May-2 June 2010 ,Paris, France, pp. 253-256, IEEE, 10.1109/ISCAS.2010.5537907.

Liang, Z., Powell, A., Ersoy, I., Poostchi, M., Silamut, K., Palaniappan, K., et.al, CNN-based image analysis for malaria diagnosis, In 2016 IEEE International Conference on Bioinformatics and Biomedicine (BIBM), 15-18 December 2016, Shenzhen, China, pp. 493-496, IEEE.

Lin, Y., Lv, F., Zhu, S., Yang, M., Cour, T., Yu, K., ... \& Huang, T., Large-scale image classification: fast feature extraction and svm training, In CVPR 2011, June 2011, pp. 16891696, IEEE.Moon, S., Lee, S., Kim, H., FreitasJunior, L. H., Kang, M., Ayong, L., \& Hansen, M. A., An image analysis algorithm for malaria parasite stage classification and viability quantification. PloS one, 8(2013), e61812.

Nugroho, H. A., Akbar, S. A., \& Murhandarwati, E. E. H., Feature extraction and classification for detection malaria parasites in thin blood smear, In 2015 2nd International Conference on Information Technology, Computer, and Electrical Engineering (ICITACEE), October 2015, Indonesia, pp.197-201, IEEE.
Poostchi, M., Silamut, K., Maude, R. J., Jaeger, S., Thoma, G., Image analysis and machine learning for detecting malaria, Translational Research, 194 (2018), pp.36-55.

Prasad, K., Winter, J., Bhat, U. M., Acharya, R. V., \& Prabhu, G. K., Image analysis approach for development of a decision support system for detection of malaria parasites in thin blood smear images. Journal of digital imaging, 25 (2012), pp.542-549.

Ring, M., Eskofier, B. M., An approximation of the Gaussian RBF kernel for efficient classification with SVMs, Pattern Recognition Letters, 84(2016), pp.107-113.

U.S. National Library of MedicineLister Hill National Center for Biomedical Communications, Accessed on (https://lhncbc.nlm.nih.gov/publication/pub9932)

World Health Organisation, https://www.who.int/malaria/areas/diagnosis/en/ "MalariaDiagnostic testing" 